\title{
Coupling and Coordination Measurement of the Development Level of China's Financial Industry and Logistics Industry
}

\author{
Qiqing Zhou ${ }^{1}$,Yuhan $\mathrm{Kong}^{2}$,Panpan Yin ${ }^{3}$ \\ ${ }^{1}$ Shaanxi Institute of International Trade \& Commerce Xi'an, China \\ ${ }^{2}$ Northwest University Xi'an, China \\ ${ }^{3}$ South-Central University for Nationalities Wuhan, China
}

\begin{abstract}
Based on the panel data of 31 China's provinces and cities, depending on discussing the significance of the coordination development of the financial industry and the logistics industry, the entropy method and the coupling coordination degree model were used to empirically analyze the dynamic coupling development of China's financial industry and logistics industry. Empirical analysis shows that during the sample period, the coordination development level of China's logistics industry and the financial industry had achieved a transition from overall disorder to overall coordination, but there was still a large potential for improvement. The level of coupling and coordination development was east-central-west declining trend in three steps. Guangdong was the province with the highest level of coupling and coordination development. Tibet, Qinghai, Ningxia, Hainan, and Tianjin were the provinces and cities with the lowest level of coupling and coordination development.
\end{abstract}

\section{Introduction}

When the wheel of history has entered the second decade of the 21st century, China's financial institutions have entered a period of the new normal development. With the increasing trend of deposit financing and the rapid development of Internet finance, the low-cost funds of domestic commercial banks continue to be diverged. At the same time, as the reform entered the deep-water zone, a large number of foreign banks poured into the country, and private banks began to appear. Internet finance business launched an impact on traditional finance. Bank profit margins were significantly narrowed, and offbalance-sheet activities risks increased sharply. Under the circumstances, the competition among commercial banks has become fierce, and the scope of traditional financing business has become a red sea. Therefore, it has become the consensus and development direction of more and more commercial banks to improve their own innovation capabilities and explore financial innovation products with high risk premiums. At the same time, China's logistics industry has entered a new stage of medium to high speed growth of about $10 \%$ after experiencing a high growth stage of $20 \%$ during $1990-2000$ and $15 \%$ during 2001 2010. At present, the logistics industry has fully entered the red sea era. The profit margins of basic logistics businesses such as warehousing and transportation are declining. The logistics industry is also in urgent need of finding a new profit growth point. The theory of industrial integration points out the direction for the development of the financial industry and logistics industry. Promoting the coupling and coordination development of the financial industry and the logistics industry can solve the problems they are facing, and ultimately bring about a win-win result.

\section{Literature Review}

Analyzed the significance of the coupling and coordination development of the financial industry and the logistics industry from a theoretical perspective. Zhu Hanmin (2003) pointed out that the development of the financial industry has a guarantee, support, supervision, and promotion effect on the development of modern logistics. The financial industry and modern logistics should interact and develop with information as a link. ${ }^{[1]}$ Liu Xiaochun and Peng Zhizhong (2009) pointed out that the coupling and coordination development of the financial industry and the logistics industry promoted the birth of the logistics financial business, thereby creating a new profit growth point for the financial industry and the logistics industry, and also provides a new solution for the financing problems of small and medium-sized enterprises. ${ }^{[2]}$ Liu Yetian and Peng Zhizhong (2009) pointed out that the coordination development of the financial industry and the logistics industry has positive significance in reducing logistics service costs, improving logistics service quality, optimizing financial risk control, and helping to solve the financing difficulties of small and medium-sized enterprises. ${ }^{[3]}$

Used the econometric model to test whether there is a coordination development relationship between finance and logistics. Li Xinguang and Zhang Yongqi (2013) used 
the SVAR model and other econometric models to empirically analyze the coordination development of finance and logistics in Fujian Province, and found that the development level of the insurance industry and the scale of the financial industry are both Granger reasons for the development level of the logistics industry. At the same time, the impact of insurance industry and the scale of financial development on the development level of the logistics industry has to go through a short period of incubation and then, it will have a positive impact on the logistics industry. ${ }^{[4]}$ Chen Ling (2014) used the VAR model to study the financial data and logistics data of Henan Province from 1993 to 2012 and found that increasing the financial breadth can have a long-term positive effect on the logistics industry. The logistics industry will restrict financial breadth and financial depth in a short term, but it will produce promotion in the long run. [5]

To measure the level of coupling and coordination development, Zhang Jianjun and Zhao Qilan (2018) adopted a dynamic coupling coordination degree model method based on entropy weights and found that the coordination between China's logistics industry and the financial industry has undergone a process of change from incoordination to barely coordination to coordination. ${ }^{[6]}$ Chu Xuejian and Qian Sainan (2019) found that the level of coupling coordination between China's logistics industry and the financial industry has been rising continuously based on the method of coupling coordination degree and gray correlation degree and has undergone a state change from disorder to good coordination. Railway operation mileage, the number of people employed in the logistics industry, the input-output ratio of the logistics industry, the scale of social financing, and the loan-to-deposit ratio are key factors in the interaction between the logistics industry and the financial industry. ${ }^{[7]}$

To sum up, scholars have done many researches on finance and financial coupling and coordination development and have achieved fruitful results. But there are also some shortcomings. The innovation of this research is mainly reflected in two aspects. The first is to construct a financial subsystem evaluation system from the three dimensions of scale, structure, and efficiency. The financial scale is expressed in terms of stock transaction volume, FIR, and insurance depth to ensure the financial subsystem evaluation system more complete, and its measurement results are more reflective of China's level of coupling and coordination development of the financial industry and the logistics industry. The second is to use the panel data of 31 provinces and cities across the country to measure the level of coupling and coordination development of the financial industry and the logistics industry, while previous scholars mostly used time series data.

\section{The Coupling and Coordination Development Status of China's Financial Industry and Logistics Industry}

Referred to the practices of most scholars such as $\mathrm{Li}$ Xinguang, Zhang Yongqi (2013) and Chen Ling (2014) ${ }^{\text {[4]- }}$ [5] we selected the added value of transportation warehousing and the postal industry and the volume of goods turnover as the measurement indicators from the two dimensions of supply and demand. Indicators are used as measurement indicators for the logistics industry. Financial correlation rate and financial structure were selected as the measurement indicators of the financial industry from the three dimensions of scale, efficiency, and structure. The financial structure indicators were based on the practice of Liu Guanchun (2017) ${ }^{[8]}$. The index weights of financial subsystem and logistics subsystem adopt entropy weight method as the value method.

Adopting entropy method as the weight value method requires 6 calculation steps. The first step is to use the linear proportional transformation method (positive indicator) to standardize the data to eliminate the dimension $X_{i j t}^{*}=\frac{\mathrm{x}_{\mathrm{ijt}}}{\mathrm{MAXx}_{\mathrm{ijt}}}$. The second step is to calculate the $i$-th evaluation object under the $j$-th indicator in year $t$ : $y_{i j t}=\frac{X_{i j t}^{*}}{\sum_{i=1}^{31} \sum_{t=2004}^{2017} X_{i j t}^{*}}$. In step 3, calculate the entropy of the $j$-th index value: $E_{j}=$ $-\frac{1}{\ln (31 \times 14)} \sum_{i=1}^{31} \sum_{t=2004}^{2017} y_{i j t} \ln y_{i j t}$. In step 4, calculate the difference coefficient of the $j$-th index: $G_{j}=1-E_{j}$. In step 5, calculate the weight of the index $j: W_{j}=\frac{G_{J}}{\sum_{j=1}^{5} G_{J}}$. The last step is to calculate the comprehensive development level of a subsystem of the $i$ - $t h$ provinces and cities in year $t: L_{i t}=\sum_{j=1}^{6} W_{j} X_{i j t}^{*}$.

The indicators of China's financial subsystem and logistics subsystem, the year-end RMB deposit and loan balances and GDP data of financial institutions in various provinces and cities were derived from the statistical yearbooks of various provinces and cities. The stock transactions were from the "China Securities and Futures Statistical Yearbook", the data on the number of employees in the transportation warehousing and postal industry came from the "China Statistical Yearbook". The rest of the data were obtained from the data query section of the official website of the National Bureau of Statistics.

After substituting these relative data, the development level evaluation system of China's financial and logistics industry was obtained as shown in Table 1.

According to the researches of Zhang Jianjun, Zhao Qilan (2018), Chu Xuejian and Qian Sainan (2019) [6]-[7], the measurement model of the coupling degree between China's financial industry and logistics industry is: $\mathrm{C}=$ $2 \sqrt{\frac{W F}{(W+F)^{2}}}$.

$\mathrm{W}$ represents the development level of the logistics subsystem. F represents the development level of the financial subsystem. C represents the degree of coupling between the logistics subsystem and the financial subsystem, and the value range is $[0,1]$. 
Table1. The development level evaluation system of China's financial and logistics industry

\begin{tabular}{|c|c|c|c|}
\hline Subsystem & Criteria layer & $\begin{array}{l}\text { Indicator level } \\
\text { Transportation warehousing and postal industry added value }\end{array}$ & weight \\
\hline \multirow{5}{*}{$\begin{array}{l}\text { Logistics } \\
\text { subsystem }\end{array}$} & Logistics & (hundred million yuan) & \\
\hline & demand & $\begin{array}{l}\text { Transportation freight volume } \\
\text { Frejght ton-kilometers }\end{array}$ & $\begin{array}{l}0.167 \\
0.162\end{array}$ \\
\hline & & Transportation warehousing and postal industry fixed asset & 0.166 \\
\hline & Logistics & $\begin{array}{l}\text { investment (hundred million yuan) } \\
\text { Mileage }\end{array}$ & 0.170 \\
\hline & & $\begin{array}{l}\text { Transportation warehousing and postal industry employees } \\
\text { (person) }\end{array}$ & 0.169 \\
\hline \multirow{4}{*}{$\begin{array}{l}\text { Financial } \\
\text { subsystem }\end{array}$} & Financial & $\begin{array}{l}\text { Financial interrelations ratio } \\
\text { Insurance depth }\end{array}$ & $\begin{array}{l}0.216 \\
0.161\end{array}$ \\
\hline & & Stock transactions /GDP & 0.200 \\
\hline & $\begin{array}{l}\begin{array}{l}\text { Financial } \\
\text { structure }\end{array}\end{array}$ & Loan/Deposit & 0.218 \\
\hline & $\begin{array}{l}\text { Financial } \\
\text { efficiency }\end{array}$ & Financial structure $=$ Stock transactions /Loan & 0.205 \\
\hline
\end{tabular}

In order to avoid the extreme situation of false evaluation results when the values of $\mathrm{W}$ and $\mathrm{F}$ are small at the same time, and considering the growth and evolution levels of the two subsystems, a coupling coordination degree model is constructed: $\mathrm{T}=\alpha \mathrm{W}+\beta \mathrm{F} ; \mathrm{D}=$ $\sqrt[2]{C \times T}$

$\mathrm{T}$ represents the comprehensive evaluation indicator of the financial and logistics subsystems, $\alpha$ and $\beta$ are respectively undetermined coefficients, with a value of 0.5 . $\mathrm{D}$ represents the degree of coupling and coordination between the financial subsystem and the logistics subsystem, and the value range is $[0,1]$. Refer to Table 2 for the classification standard of coupling coordination degree.

Table2. standard on the coupling and coordination degree classification of China's financial industry and logistics industry

\begin{tabular}{|c|c|c|c|c|c|}
\hline $\begin{array}{l}\text { Coordination } \\
\text { range }\end{array}$ & $\begin{array}{l}\text { Coordination } \\
\text { degree }\end{array}$ & $\begin{array}{l}\text { Coordination } \\
\text { range }\end{array}$ & $\begin{array}{l}\text { Coordination } \\
\text { degree }\end{array}$ & $\begin{array}{l}\text { Coordination } \\
\text { range }\end{array}$ & $\begin{array}{c}\text { Coordination } \\
\text { degree }\end{array}$ \\
\hline$\left[\begin{array}{ll}0, & 0.1)\end{array}\right]$ & $\begin{array}{l}\text { Extreme } \\
\text { disorder }\end{array}$ & {$[0.4,0.5)$} & $\begin{array}{l}\text { Slightly } \\
\text { disorder }\end{array}$ & {$[0.8,0.9)$} & $\begin{array}{c}\text { High } \\
\text { coordination }\end{array}$ \\
\hline$[0.1,0.2)$ & $\begin{array}{l}\text { Serious } \\
\text { disorder }\end{array}$ & {$[0.5,0.6)$} & $\begin{array}{c}\text { Barely } \\
\text { coordination }\end{array}$ & {$[0.9,1)$} & $\begin{array}{c}\text { Extreme } \\
\text { coordination }\end{array}$ \\
\hline$[0.2,0.3)$ & $\begin{array}{l}\text { Moderate } \\
\text { disorder }\end{array}$ & {$\left[\begin{array}{ll}0.6, & 0.7)\end{array}\right.$} & $\begin{array}{l}\text { Low } \\
\text { coordination }\end{array}$ & & \\
\hline$\left[\begin{array}{lll}0 & 3 & 04\end{array}\right]$ & Low disorder & 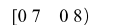 & Moderate & & \\
\hline
\end{tabular}

After substituting the data of logistics subsystem and financial subsystem into the coupling coordination degree model, Table 3 is obtained.

According to the analysis, we found that, firstly, the level of the coordination development of China's logistics industry and financial industry has made great progress in the past ten years. During the sample period, the degree of coupling and coordination between the logistics industry and the financial industry has continued to grow, achieving a transition from overall disorder to overall coordination. However, the coordination development level of China's logistics industry and financial industry is still at a low level. As of 2017, most provinces and cities across the country are still at a barely coordination development level, and even Hainan and Ningxia are still in a state of low disorder which means that there is still much potential in the level of coupling and coordination development of the financial industry and the logistics industry.

Secondly, the coordination development level of China's logistics industry and financial industry shows a declining trend from east-middle-west. The degree of coupling and coordination in the eastern provinces and cities is mostly at a low level of coordination development, the degree of coupling and coordination in the central provinces and cities is mainly at the level of barely coordination, and in the western region is uneven.
Table3. The coupling and coordination development level of

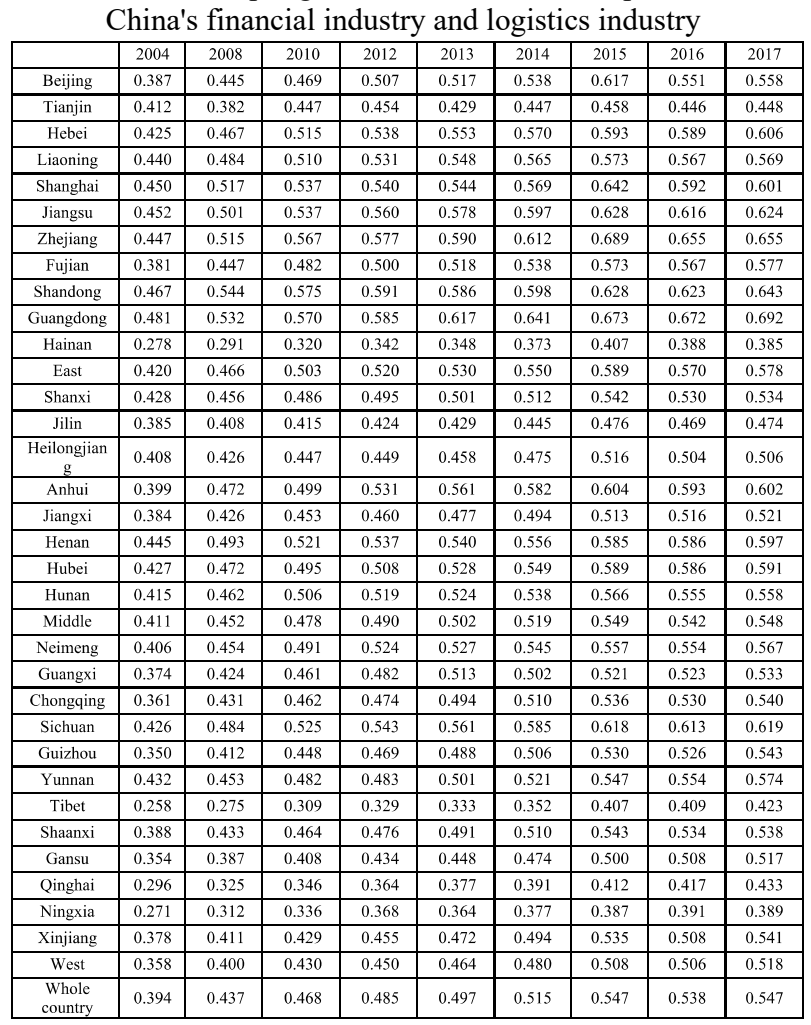

Thirdly, Guangdong is the province with the highest level of coupling and coordination development of China's logistics industry and financial industry. Since 2013, Guangdong has been in a state of moderately degree between the logistics industry and the financial industry for four consecutive years. Its degree has been increasing year by year. There are several reasons for this situation. First, with the strong support of governments at all levels. Governments at all levels in Guangdong Province have successively issued the "Guangdong Province Modern Logistics Development Plan (2016-2020)", "Guangzhou City's Implementation Opinions on Promoting the Development of Supply Chain Finance", "Opinions on Promoting the Development of Shenzhen Supply Chain Finance" and a series of documents have strongly supported the development of supply chain finance, logistics finance and other businesses. Furthermore, Guangdong's economy is developed, with a large number of manufacturing centers such as Shekou, Dongguan, and Foshan. In addition, Guangdong's third-party logistics is relatively developed, so there are huge Logistics development needs and the demand for providing logistics value-added services. Otherwise, Shenzhen is the vanguard of China's reform and opening up. New financial formats such as supply chain finance have developed very early and are showing an increasingly development trend. The Shenzhen Development Bank began to provide " $1+\mathrm{N}$ " supply chain financial services as early as 2003 and issued a supporting management system such as the "Administrative Measures for the Establishment of Branch Trade Finance Business Institutions". After being acquired by Ping'an company, it launched the "Orange eplatform", closely linked to all links in the trade process and provided powerful financing service payments for upstream and downstream customers in the industry chain. 
Fourthly, Tibet, Qinghai, Ningxia, Hainan, and Tianjin are the provinces with the lowest level of coupling and coordination development of China's logistics industry and financial industry. We believe that the main reason lies in the unbalanced development of logistics subsystems and financial subsystems in these provinces and cities. If the ratio of the development level of the logistics subsystem $\mathrm{W}$ to the development level $\mathrm{F}$ of the financial subsystem is used to measure the leading or lagging degree of the logistics subsystem relative to the financial subsystem, when the ratio is greater than 1 , the logistics subsystem is ahead of the financial subsystem. When the ratio is less than 1, the opposite is true and the ratio is equal to 1 , which means the two develop simultaneously. Through calculations, we can know that the logistics subsystems of Tibet, Qinghai, Ningxia, Hainan, and Tianjin are far ahead of the development of the financial subsystem.

\section{Conclusions and Suggestions}

\subsection{Making new policies for eastern region}

Through empirical analysis, it can be clarified that the policies formulated after 2008 have played a positive role in improving the coordination development of China's financial industry and logistics industry. The eastern region is the region with the highest level of coordination development between the financial industry and the logistics industry in China. The coordination development level of the financial industry and logistics industry in Shanghai, Zhejiang, Jiangsu, Guangdong and other provinces and cities has entered a low level of coordination. Therefore, it is necessary to comb and summarize systematically on the "Medium and Long-term Plan for the Development of the Logistics Industry (20142020)", "Guiding Opinions of the General Office of the State Council on Actively Promoting Supply Chain Innovation and Application", and "Guangzhou City's Implementation Opinions on Promoting the Development of Supply Chain Finance" and other documents, and then combined with the current economic operating environment, targeted new policies to further improve the coordination development of China's financial industry and logistics industry.

\subsection{Differentiate to solve the problem of coordination development of financial industry and logistics industry in various regions}

The level of coupling and coordination development of the financial industry and the logistics industry in various areas is inconsistent, and the reasons is also inconsistent, which need to be treated differently. Due to differences in geographic factors, industrial support and leading industries, the development level of logistics subsystems in Tibet, Qinghai, Ningxia, Hainan, and Tianjin is much higher than that of financial subsystems. Therefore, it is necessary to improve the coordination development level of these two industries in areas above mentioned. It has to accelerate the development of the logistics industry. on the contrary, for Shandong, Liaoning, Shaanxi and other provinces and cities, the development of the financial subsystem needs to be accelerated.

\subsection{Constructing a horizontal coordination mechanism between provinces}

The core of the idea of unbalanced development is to first realize the priority development of a certain area, and then drive the development of the late-developing areas through the developed areas. At present, the level of coordination development of the financial industry and logistics industry in eastern China is higher than that in other regions. The coordination development level of the financial industry and logistics industry in Jiangsu, Zhejiang, Guangdong and other provinces has entered a low-level coordination stage, so it is necessary to establish build a horizontal coordination mechanism among the provinces to strengthen the strategic interaction of the eastern, central and western provinces, to promote the free flow of financial and logistics elements between provinces. Furthermore, sufficient using the radiation and driving capabilities of provinces with high levels of coupling and coordination development such as Zhejiang and Guangdong, forming the good mutual promotion situation in the eastern region drive the central and western regions, and the central and western regions vigorously catch up the eastern region.

\subsection{Focusing on solving the problem of the coordination development of the financial industry and the logistics industry in disadvantaged areas}

To improve the level of coupling and coordination development level of China's financial industry and logistics industry, we must pay special attention to and treat weak areas such as Hainan, Tianjin, Qinghai, Ningxia, and Tibet. Specifically, the following measures can be taken. One is to use provinces with high levels of coupling and coordination development of financial and logistics industries such as Guangdong, Zhejiang, and Sichuan as benchmarks to learn from the advanced experience and excellent practices of these provinces. The second is to take advantage of the "One Road" initiative, the construction of China (Hainan) Free Trade Pilot Zone and other policies. The third is to establish an inter-provincial horizontal coordination mechanism with neighboring provinces such as Guangdong to guide the financial and logistics resources of these well-developed regions to Hainan, Tianjin, Qinghai, Ningxia, Tibet and other regions. The fourth is to provide convenience in business innovation, enterprise introduction, and talent introduction.

\section{Acknowledgment}

We thank for the support from Research on Internet Data Property Rights (2018F028) of Shaanxi Social Science Fund Project and Research on Financial Support in Shaanxi Rural Revitalization Strategy (GJM201906) of 2019 Key Bidding Project, Buchang Xixian Economic 
Research Institute, Shaanxi Institute of International Trade \& Commerce.

Research on Internet Data Property Rights (2018F028) of Shaanxi Social Science Fund Project.

Research on Financial Support in Shaanxi Rural Revitalization Strategy (GJM201906) of 2019 Key Bidding Project, Buchang Xixian Economic Research Institute, Shaanxi Institute of International Trade \& Commerce.

\section{References}

1. Zhu Hanmin. The interactive development of my country's financial industry and modern logistics [J]. Journal of Wuhan University of Technology (Social Science Edition), 2003 (03): 226-230.

2. Liu Xiaochun, Peng Zhizhong. Research on the application of coordinated development of logistics and finance $[\mathrm{J}]$. China Circulation Economy, 2009 (04): 23-25.

3. Liu Yetian, Peng Zhizhong. Research on the Construction of Coordinated Development Mechanism of Logistics Industry and Financial Industry [J]. Dongyueluncong, 2009 (06): 38-41.

4. Li Xinguang, Zhang Yongqi. Research on the Interactive Relevance of Financial Support to the Development of the Logistics Industry Based on the SVAR Model-Taking Fujian Province as an Example [J]. Journal of Shandong University of Technology (Social Science Edition), 2013 (04): 1521.

5. Chen Ling. Research on the collaborative development mechanism of regional finance and logistics_ A full-perspective analysis based on the SVAR model [J]. Economic Issues, 2014 (01): 49$52+57$.

6. Zhang Jianjun, Zhao Qilan. Research on the coordination development of China's logistics industry and financial industry based on canonical correlation analysis and coupling coordination degree [J]. Industrial Technology Economy, 2018 (08): 1219.

7. Chu Xuejian, Qian Sainan. Research on the coordination development of China's logistics industry and financial industry based on the degree of coupling coordination and the degree of gray correlation [J]. Industrial Technology Economy, 2019 (07): 93-100.

8. Liu Guanchun. The financial structure influences the transmission mechanism of the urban-rural income gap _ A study based on the dual perspectives of economic growth and urbanization $[\mathrm{J}]$. Finance, Trade and Economics, 2017 (06): 98-114. 